\title{
Metastatic ovarian cancer to the gallbladder indicative of the diagnosis
}

\begin{abstract}
Ovarian cancer is a malignant tumor that usually develops from the surface coating of the ovaries. The most common form is epithelial carcinoma. As a result of its location, and its silent nature responsible for a delay in diagnosis that makes the prognosis rather poor. The usual metastatic sites are the peritoneal cavity, liver and lung. Secondary biliary localization is a rare, even exceptional site. We report the observation of a patient who presented with abdominal pain in the right hypochondrium and progressive vomiting. Abdominal ultrasound revealed a $21 \mathrm{~mm}$ gallbladder lithiasis with hepatic steatosis. Abdominal CT scan revealed a large heterogeneous mass with engulfed gallstones. The patient underwent cholecystectomy. Histological study showed moderately differentiated adenocarcinoma and acute cholecystitis. Immunohistochemical staining revealed that the tumor cells were positive for antibodies against CK7, WT1, PAX8 and p53 and negative for CK20 and ER. These results suggest that the tumor was a metastasis of serous ovarian adenocarcinoma. Medical imaging done with abdominal CT showed an ovarian mass with peritoneal carcinosis, serum CA125 was elevated at 97U/ml. Carbohydrate antigen (CA) 19-9 and carcinoembryonic antigen (CEA) levels were normal. The patient received firstline chemotherapy with carboplatin and paclitaxel. After 6 courses of therapy, she achieved a partial clinical, biological and radiological response, in our medical oncology department of the CHU Hassan II from Fez.
\end{abstract}

The interest of the subject is to report the rarity of this entity as well as the radiological, histopathological, prognostic and therapeutic characteristics.
Volume II Issue I - 202 I

\section{Soumia Berrad, Zineb Benbrahim,Vincent W Lokonga, Lamiae Nouiakh, Hayat Erraichi, Lamiae Amaadour, Karima Oualla, Samia Arifi, Naoufal Mellas \\ Department of Medical Oncology, Hassan II Unversity Hospital, Morocco}

Correspondence: Soumia Berrad, Department of Medical Oncology, Hassan II Unversity Hospital, Morocco,

Email soumiaberrad3@gmail.com

Received: December 20, 2020 | Published: January 25, 2021

Keywords: ovarian cancer, metastatic, gallbladder, chemotherapy

\section{Introduction}

Ovarian cancer is the fourth most common gyneco-mammary cancer in women with an incidence of $3.4 \% .{ }^{1}$ With a very high prevalence in industrialized countries, it mainly affects women in the postmenopausal period and represents the fourth leading cause of female death from cancer.

In Morocco, ovarian cancer accounts for about $4 \%$ of female cancers, responsible for more than $5 \%$ of cancer deaths. The incidence and mortality rates tend to decrease with the progress of treatment. ${ }^{2}$

Its peculiarity is based on its silent nature responsible for delayed diagnosis and therapeutic difficulty, especially in its extensive forms.

Ovarian cancer can metastasize through the peritoneal, bloodstream, or lymphatic route. The most common sites of metastasis are the peritoneum, abdominal lymph nodes, liver and lung. ${ }^{3}$

Some studies have reported that cancer of the The ovary may present as obstructive jaundice by invasion of the extrahepatic bile duct by perhepatichilar lymphadenopathy. ${ }^{4}$

Four metastatic modes of dissemination are described in ovarian cancer. Direct invasion by dissemination to adjacent structures, Lymphatic invasion, and In most cases, this invasion is peritoneal begins with infiltration of the serosa, however Haematogenous dissemination is rare. ${ }^{5}$ Vesicular metastases indicative of a diagnosis of ovarian cancer are extremely rare.

We report an observation of a 57-year-old patient diagnosed with ovarian cancer secondary to gallbladder localization in the medical oncology department of CHU HASSAN II in Fez.

\section{Observation}

This is a 52-year-old postmenopausal woman who presented with abdominal pain in the right hypochondrium with progressive vomiting.

Abdominal ultrasound found a $21 \mathrm{~mm}$ gallbladder lithiasis with hepatic steatosis. Abdominal CT scan revealed a large heterogenous masse with engulfed gallstones. The patient underwent a cholecystectomy.

Macroscopically the gall bladder measured $8 \times 3.5 \mathrm{cmand}$ encountered small nodule of $0.3 \mathrm{~cm}$. The histological study showed a moderately differentiated adenocarcinoma and acute cholecystitis. Immunohistochemistry staining revealed that tumor cells were positive for anti CK7, WT1, PAX8 and p53 antibodies and negative for CK20 and ER. These results suggest that the tumor was a metastasis from serous ovarian adenocarcinoma.

The abdomino-pelvic scanner showed peritoneal carcinomatosis secondary to a right ovarian mass without digestive thickening or deep adenopathy and without bone abnormalities. We completed with pelvic ultrasound showing a par-uterine mass measuring $81 \mathrm{~mm}$ with great abundance effusion.

The serum level of CA125 was high at 97U/ml. Carbohydrate antigen (CA) 19-9 and carcinoembryonic antigen (CEA) levels were normal.

The case was discussed in the tumor board meeting and retained gallbladder metastasis and peritoneal carcinomatosis from high grade serous ovarian cancer. 
The patient received first line chemotherapy based on carboplatin and paclitaxel. After 6 cures she obtained a partial clinical, biological and radiological response (Figure 1).

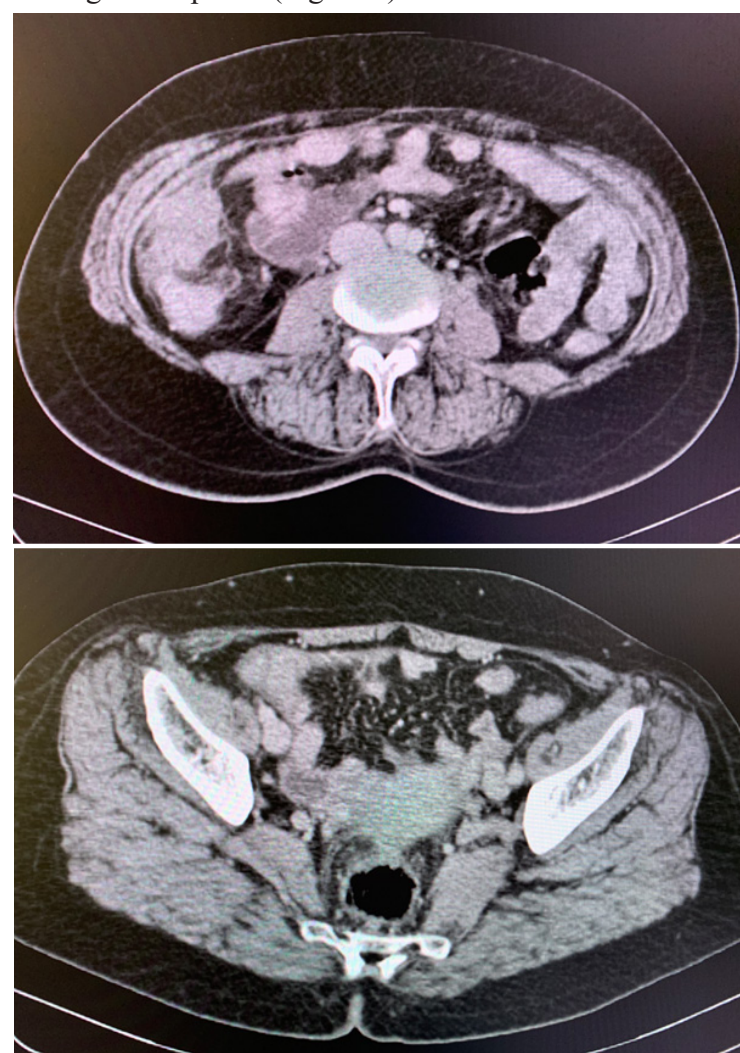

Figure I (a) (b) The abdomino-pelvic scanner showed peritoneal carcinomatosis secondary to a right ovarian mass without digestive thickening or deep adenopathy and without bone abnormalities.

\section{Discussion}

Ovarian cancer is a disease that is asymptomatic for a long time, making early clinical diagnosis difficult and the prognosis of this pathology quite pejorative. ${ }^{5}$

The metastatic spread of ovarian cancer occurs via the hematogenous lymphatic or transperitoneal route which is the main mode of spread by detachment of malignant cells in the peritoneal cavity. ${ }^{6}$

Intraperitoneal propagation is also favored by intestinal peristalsis, negative hydrostatic pressure under the diaphragm. ${ }^{7}$

However, the peritoneal cavity is the site most often involved in $83 \%$ to $100 \%$, then lymph node in $50 \%$ to $60 \%$ of cases, followed by the intestine, liver and lung. ${ }^{8}$

The gallbladder is a very rare site of ovarian cancer metastasis It can be invaded either by its wall, or by contiguous masses or by lymph nodes. ${ }^{9}$

Among the cancers listed metastases to the biliary level are primary cancers of the lung, breast, gallbladder, colon, testes, prostate, pancreas, melanoma and lymphoma. Metastases from Ovarian adenocarcinoma are rarely reported in the literature.

The metastatic lesions appear hyper vascularized on the abdominal injected CT scan. In the present case, hypervascularization was objectived with gallstones.
Immunohistochemical staining plays a crucial role in the histological confirmation of the diagnosis. In this patient, it revealed positive staining WT-1 increasing the optimal treatment for metastatic ovarian cancer at the biliary level with multiple metastatic sites would be chemotherapy. In general, the combination of platinum salts of carboplatin, paclitaxel and bevacizumab is considered to be standard in advanced ovarian cancer. ${ }^{10}$

Meta-analysis published in 2002 including the 4 trials ,GOG111, OV-10, GOG132, ICON 3, showed a benefit of the combination of paclitaxel-based chemotherapy - platinum, ${ }^{11} 2004$ consensus statement on the management of ovarien cancer allowed the adoption of paclitaxel carboplatin as standard first-line treatment in metastatic ovarian cancer. ${ }^{12}$

The SCOTROC scotishpahse III randomized trial comparing of paclitaxel-carboplatin AUC5 versus dopcetaxelcarboplatine as first line chemotherapy for ovarien cancer, demonstrated a similar efficacy in terms of PFS and response, neurosensory toxicity was more marked with paclitaxel while haematological toxicity was statistically higher in the docetaxel arm. ${ }^{13}$

Several studies haves evaluated the activity of bevacizumab when combined to chemotherapy in ovariancancer,ICON7, GOG 218 Two phase III randomized trials showed that the addition of bevacizumab improved progression-free survival in women with ovarian cancer. The benefits in terms of progression-free and overall survival were greater in people at high risk for disease progression. ${ }^{14,15}$

Hepatectomy with caudate lobectomy with extrahepaticbil duct can be offered in the presence of a single biliary metastasis. ${ }^{16}$

\section{Conclusion}

Ovarian cancer remains a heterogeneous pathology, which remains for a long time unsymptomatic and with a poor prognosis. It is a rare case of ovarian metastases in the gallbladder, revealing the diagnosis. A correct diagnosis may be possible after a complete clinical evaluation and radiological investigation combined with detailed histological and molecular analysis. Biliary metastases of ovarian cancer often indicate an advanced stage of the disease with a poor prognosis, where treatment with chemotherapy and biotherapy is indicated.

\section{Conflicts of interest}

The author declares no conflict of interest.

\section{Acknowledgments}

None.

\section{Funding}

None.

\section{References}

1. Freddie Bray, Jacques Ferlay, Isabelle Soerjomataram, et al. Global cancer statistics 2018: GLOBOCAN estimates of incidence and mortality world wide for 36 cancers in 185 countries. C A Cancer J Clin. 2018;68(6):394 424.

2. Mustapha Benhessou, Luc MagloireAnicet Boumba, Mohammed Nabil Benchkrroun, et al. Epithelial cancer of the ovary in Morocco: Epithelial analysis of a series of 182 cases at the CHU Ibn Rochd in Casablanca, Maroc International Journal of Innovation and Scientific Research. 2016;20(1):153-162. 
3. Güth U, Huang DJ, Bauer G, et al. Metastatic profiles at autopsy in patients with ovarian carcinoma. Cancer. 2007;110(6):1272-1280.

4. Rosenblatt M, Zafaranloo S, Tancer ML. Carcinoma of the ovary presenting as obstructive jaundice. Gynecol Oncol. 1989;32(3):385-389.

5. JH Bijek, N Ehnart, P Mathevet, et al. Dissémination métastatique par voie hématogène d'un cancer épithélial de l'ovaire : à propos d'un cas Hematogenous dissemination in epithelial ovarian cancer: Case report. Journal de Gynécologie Obstétrique et Biologie de la Reproduction. 2011;40(5):465-468.

6. Lansac J, Bonnamy L. Clinical presentation of benign and malignant ovarian tumors. Encycl Med Chir, Scientific and Medical Editions Elsevier SAS, Paris. Gynecology. 2000;630-D-10.

7. De Vita. Vincent T. Lawrence, Theodore S. Principles and Practice of Oncology. 8th edn, 2008.

8. Halperin EC. Perez, Carlos A. Perez and Brady Principles and Practice of Radiation Oncology, 5th edition, 2008.

9. Uwe Güth, Dorothy Jane Huang, Gregor Bauer, et al. Metastatic profiles at autopsy in patients with ovarian carcinoma. Cancer. 2007;110(6):12721280 .

10. A prospective, randomized phase III study to evaluate the optimal duration of treatment with first-line bevacizumab in combination with carboplatin and paclitaxel in patients with primary epithelial cancer of the ovary and tube. Fallopian or peritoneum.
11. J Sandercock, MKB Parmar, V Torri, et al. First-line treatment for advanced ovarian cancer: paclitaxel, platinum and the evidence. $\mathrm{Br} J$ Cancer. 2002;87(8):815-824.

12. A du Bois, M Quinn T Thigpen, J Vermorken, et al. 2004 sensus statements on the management of ovariancancer: final document of the 3rd International Gynecologic Cancer IntergroupOvarian Cancer Consensus Conference (GCIG OCCC 2004). Ann Oncol. 2005;viii7-viii12.

13. Paul A Vasey, Gordon C Jayson, Alan Gordon, et al. Phase III Randomized Trial of Docetaxel-Carboplatin Versus Paclitaxel-Carboplatin as First-line Chemotherapy for Ovarian Carcinoma. J Natl Cancer Inst. 2004;96(22):1682-1691.

14. Timothy J Perren, Ann Marie Swart, Jacobus Pfisterer, et al. A Phase 3 Trial of Bevacizumab in Ovarian Cancer. N Engl J Med. 2011;365(26):24842496.

15. Burger RA, Brady MF, Bookman MA, Incorporation of bevacizumab in the primary treatment of ovarian cancer. Engl J Med. 2011;365(26):24732483.

16. MasahiroShijo, Koji Fukase, HideoOhtsuka, et al. Metastasis of ovarian cancer to the bile duct: a case report. Surg Case Rep. 2019;5(1):100. 